\title{
Antioxidant and Phenolic Changes Across One Harvest Season and Two Storage Conditions in Primocane Raspberries (Rubus idaeus L.) Grown in a Hot, Dry Climate
}

\author{
Brenner L. Freeman \\ Department of Nutrition, Dietetics and Food Science, Brigham Young \\ University, 685 E University Pkwy, S-135 ESC, Provo, UT 84602
}

Janet C. Stocks

Cornaby Farms, 671 W Salem Canal Road, Salem, UT 84664

Dennis L. Eggett
Department of Statistics, Brigham Young University, 685 E University Pkwy,
223 A TMCB, Provo, UT 84602

Tory L. Parker ${ }^{1}$

Department of Nutrition, Dietetics and Food Science, Brigham Young University, 685 E University Pkwy, S-135 ESC, Provo, UT 84602

Additional index words. raspberry, ORAC, total phenolics, primocane, fertigation

\begin{abstract}
Antioxidant phytochemical differences among cultivars and changes after storage have not been explored in primocane raspberries grown in a hot, dry climate. It was hypothesized that significant differences would be detected in total phenolics and antioxidant capacity [oxygen radical absorbance capacity (ORAC)] between cultivars and over time. 'Autumn Bliss' had the highest ORAC and phenolic content, whereas 'Caroline' had the lowest ORAC and phenolic content when fresh. Averaging all cultivars and pickings, refrigerated berries had a significantly higher total phenolic content than fresh or frozen berries, although some moisture loss occurred during standard refrigeration. Antioxidant capacity and total phenolic content of primocane raspberries grown in hot, dry conditions vary between cultivars, on average do not change significantly after storage, are higher later in the season, and are higher than supermarket cultivars.
\end{abstract}

Fruit and vegetable consumption has been associated with decreased mortality and lowers the incidence of cancer (Dragsted et al., 1993) and cardiovascular disease (Verlangieri et al., 1985). Fruits contain several different families of phytochemicals, which are thought to contribute to this decreased risk. Antioxidant capacity is one measure of phytochemical content. Those compounds responsible for most of a fruit's antioxidant capacity are phenolic acids, anthocyanins, and other flavonoids (Cao et al., 1997). Antioxidant capacity, anthocyanin content, and total phenolic content are highest when a fruit is ripe (Wang and Lin, 2000). Ripe raspberries have a high antioxidant capacity and total phenolic content compared with most other berries and are a good source of ellagic acid, an antioxidant with powerful antimicro-

Received for publication 13 Sept. 2010. Accepted for publication 15 Nov. 2010.

This work was funded by the Utah Department of Agriculture and Food Specialty Crops Block Grant Program. Raspberries were provided by Cornaby Farms, Salem, UT.

${ }^{1}$ To whom reprint requests should be addressed; e-mail tory_parker@byu.edu. ods result in differences in antioxidant activity and phenolic content of raspberries (Ozgen et al., 2008). Previously published data (Anttonen and Karjalainen, 2005; De Ancos et al., 2000; González et al., 2003; Kähkönen et al., 2001; Liu et al., 2002; Ozgen et al., 2008) has limited application to dry condition raspberry farming. The objective of this study was to measure antioxidant capacity and total phenolic content of six cultivars of primocane raspberries grown under controlled fertigation (fertilizer provided to the plant through irrigation water) in Utah. It was hypothesized that antioxidant capacity and phenolic content would vary across cultivar, across the season, and between storage treatments.

\section{Materials and Methods}

Raspberry collection. Six cultivars of Rubus idaeus L. raspberries ('Autumn Bliss', 'Caroline', 'Jaclyn', 'Joan J', 'Polana', and 'Polka') grown on a 20 acre farm in Utah were collected every 2 weeks beginning 15 Aug. 2009 and ending 10 Oct. 2009 (five pickings). As the season was coming to a close, there were insufficient berries on 10 Oct. to store, so only the fresh analysis was possible. Agricultural methods used on this farm were consistent between cultivars and included raised beds (Wright et al., 2001), underground fertigation (Gurovich, 2008), and a trellis system (Handley, 2006). Only ripe red raspberries easily removed from the stem were collected; no other post-harvest treatments were performed. For most pickings, sufficient berries were picked from each cultivar to perform all analyses. Fresh berries were analyzed within $24 \mathrm{~h}$ of picking, refrigerated samples were stored in small "clam shell" plastic containers commonly used for commercial sale for $7 \mathrm{~d}$ in a standard refrigerator, and frozen berries were stored in zip seal freezer bags at $-20{ }^{\circ} \mathrm{C}$ for 3 months without initial liquid nitrogen freezing to mimic consumer freezing. $\mathrm{pH}$ and soluble solids were measured on the first two pickings of fresh berries for general comparison with literature values. ORAC and total phenolic analyses were performed on the fresh, refrigerated, and frozen berries from all pickings. Refrigerated and frozen berries were allowed to warm to room temperature before analysis.

Raspberry extraction. For ORAC and total phenolic measurements, raspberries $(100 \mathrm{~g})$ of each cultivar were blended with $100 \mathrm{~mL}$ of double deionized water $\left(\mathrm{ddH}_{2} \mathrm{O}\right)$ in a Waring commercial laboratory blender (Waring Products Inc., Torrington, CT) on high speed for 1 $\min$. One gram of raspberry puree was transferred to a $1.5-\mathrm{mL}$ centrifuge tube and centrifuged (10,000 g, 2 min; Eppendorf N.A., Hauppauge, NY). The supernatant was transferred to a $15-\mathrm{mL}$ centrifuge tube. One milliliter of 70:29.5:0.5 acetone: $\mathrm{ddH}_{2} \mathrm{O}$ :acetic acid (AWA) was then added to the raspberry pellet and vortexed $(30 \mathrm{~s}, 3000 \mathrm{rpm}$; Fisher Scientific digital vortex mixer; Fisher Scientific, Fair Lawn, NJ) to ensure a complete blend of raspberry pulp and AWA. The $1.5-\mathrm{mL}$ centrifuge tube was then sonicated ( $2 \mathrm{~min}$, $37{ }^{\circ} \mathrm{C}$; Fisher Scientific ultrasound cleaner) 
and again centrifuged $(10,000 \mathrm{~g}, 2 \mathrm{~min})$. The supernatant was added to the $15-\mathrm{mL}$ centrifuge tube. This process was repeated a second time for a total of $\approx 3 \mathrm{~mL}$ of extract in the $15-\mathrm{mL}$ tube. This process was completed for all raspberry samples in triplicate. Before use in the ORAC and total phenolics assays, all $15-\mathrm{mL}$ tubes containing extracts were again centrifuged (10,000 rpm, $2 \mathrm{~min}$ ) and samples were drawn from the supernatant.

pH and ${ }^{\circ}$ Brix. $\mathrm{pH}$ was measured immediately after picking with a Jenco $6230 \mathrm{~N} \mathrm{pH}$ meter (Jenco Instruments, San Diego, CA) after mashing $\approx 20$ fresh berries of each cultivar. Sugar content $\left({ }^{\circ}\right.$ Brix) was measured with an Atago PAL-1 refractometer (Atago U.S.A. Inc., Bellevue, WA) using the same sample prepared for $\mathrm{pH}$ measurement.

Oxygen radical absorbance capacity. The ORAC assay was performed according to Davalos et al. (2004) with some modifications. Briefly, fluorescein (Fisher Scientific) was diluted in phosphate buffer to $70.3 \mathrm{~mm}$ and stored in $25-\mathrm{mL}$ aliquots for no more than 1 month at $-20{ }^{\circ} \mathrm{C}$. Trolox (Fisher Scientific) was diluted to $5 \mu \mathrm{M}, 10 \mu \mathrm{M}, 20 \mu \mathrm{M}$, and $40 \mu \mathrm{M}$ concentrations in AWA and stored at $-20{ }^{\circ} \mathrm{C}$ in aliquots of $100 \mu \mathrm{L}$ for no more than 1 month. AAPH [2,2'-azobis(2-amidino-propane)dihydrochloride, Wako Chemical, Richmond, VA] was diluted to $12 \mathrm{~mm}$ in warm $\left(40{ }^{\circ} \mathrm{C}\right)$ phosphate buffer $5 \mathrm{~min}$ before each ORAC assay. Warm $\left(40^{\circ} \mathrm{C}\right)$ fluorescein and AAPH solutions were transferred to wells of Corning Costar 96-well black side clear bottom plates (Fisher Scientific) through a Precision Micropipettor (BioTek Instruments, Inc., Winooski, VT). All concentrations of Trolox $(5 \mu \mathrm{M}, 10 \mu \mathrm{M}, 20$ $\mu \mathrm{M}, 40 \mu \mathrm{M})$ were transferred in duplicate wells within the same row to form a standard curve. Raspberry extracts were diluted and transferred to wells in duplicate according to a predesigned plate layout. All filled plates were warmed within the plate reader (set at $37^{\circ} \mathrm{C}$ ) for $15 \mathrm{~min}$ before the addition of AAPH and subsequent fluorescence measurement. Each mirrored duplicate was averaged and counted as one replicate. All samples were measured in triplicate (six wells total).

Fluorescence of all wells was measured at $485 / 20 \mathrm{~nm}$ excitation and 528/20 nm emission every minute for $120 \mathrm{~min}$ in a BioTek Synergy 2 fluorescence plate reader (BioTek Instruments, Inc.). ORAC values were expressed as Trolox equivalents per gram (TE/g) of raspberry.

Total phenolics. Total phenolics were determined using the Folin-Ciocalteu method (Swain and Hillis, 1959) as follows. The final extract $(20 \mu \mathrm{L})$ as prepared previously was diluted $1: 10$, combined with $100 \mu \mathrm{L}$ of $10 \%$ (v/v) Folin-Ciocalteu phenol reagent (3 min) (Sigma Chemical, St. Louis, MO), followed by addition of $80 \mu \mathrm{L}$ of $75 \mathrm{~g} \cdot \mathrm{L}^{-1}$ sodium carbonate, and incubated $\left(45^{\circ} \mathrm{C}, 25 \mathrm{~min}\right)$. Absorbance was determined (BioTek Synergy 2) at $765 \mathrm{~nm}$. Standard curves were prepared daily from 120 to $2000 \mu \mathrm{mol} \cdot \mathrm{L}^{-1}$ gallic acid (Sigma Chemical).

Statistics. Data were analyzed using a mixed model analysis of variance with Tukey adjusted comparisons using SAS statistical analysis software (Version 9.1; SAS Institute Inc., Cary, NC). Three analyses were required to answer the questions posed. All terms that were significant $(P<0.05)$ were included in each model. Interaction terms that were not significant were removed from their respective analysis to generate accurate sErs. An $R^{2}$ value comparing ORAC and total phenolics was determined for the overall treatment effect data using Excel (Microsoft Inc., Redmond, CA).

\section{Results and Discussion}

$\mathrm{pH}$ and ${ }^{\circ}$ Brix. Both measures were performed on all six cultivars for the first two fresh pickings. $\mathrm{pH}(3.31 \pm 0.10$, mean $\pm \mathrm{SD})$ was very similar for all cultivars and across both weeks. This is nearly equivalent to the mean of $3.26 \pm$ 0.02 reported by De Ancos et al. (2000) for four cultivars. ${ }^{\circ}$ Brix was slightly more variable, with a mean \pm SD of $11.95 \pm 1.87$. De Ancos et al. (2000) reported a lower mean Brix value of $9.83 \pm 0.09$. Thus, the raspberries in the present study had a higher percent soluble solids, which could be attributed to cultivar, growing conditions, and/or climate.

Differences between cultivars and treatments. To determine if there were significant ORAC or total phenolic differences for the season as a whole, all values for pickings were averaged (Table 1). 'Caroline' and 'Autumn Bliss', with the lowest and the highest ORAC values, respectively, were significantly different from each other but not from other cultivars for both fresh and refrigerated berries. A similar pattern was seen for total phenolics, a significant difference between the lowest and highest values. There were no significant differences between any of the cultivars after freezing. There were no significant ORAC differences across the rows in Table 1 among fresh, refrigerated, and frozen berries for any cultivar. Only the Joan J cultivar had a significantly different total phenolic content and only between the refrigerated and frozen berries $(P=0.041)$. The total phenolic content of 'Joan J' changed most during refrigeration, whereas 'Autumn Bliss' decreased when they were frozen.

Both genetic and environmental variation have been reported as factors influencing raspberry chemical content (Anttonen and Karjalainen, 2005; Dvaranauskaite et al., 2006). These authors observed variations in quercetin content, total phenolics, anthocyanins, and ellagic acid between cultivars. We report variation between cultivars in antioxidant capacity and total phenolic content. We report a range of 237 to $952 \mathrm{mg}$ gallic acid equivalents (GAE)/100 $\mathrm{g}$ of total phenolics (565 mg GAE/100 g for 'Autumn Bliss'). Wang and Lin (2000) reported a range of 208 to 258 $\mathrm{mg}$ GAE/100 $\mathrm{g}$ of total phenolics for four cultivars of red raspberries (245 mg GAE/100 $\mathrm{g}$ for 'Autumn Bliss', also analyzed in the present study). Our total phenolic values are on average higher than those reported by Wang and Lin (2000). This is in part explained by extraction method (Wang and Lin pulverized and centrifuged only; no extraction), although production site, cultivar, or harvest season could also have been factors (Ozgen et al., 2008).

Overall storage effects. To determine the overall effect of fresh, refrigerated, or frozen treatment on all cultivars across the season, cultivar and pickings were grouped for the analysis (Table 2). There was no significant ORAC difference between fresh and refrigerated berries, but there was a significant difference in ORAC between refrigerated and frozen storage. For total phenolics, there was no significant difference between fresh and frozen berries, but a significant increase in total phenolics occurred during refrigerated storage. The relationship between ORAC and total phenolic data had an overall $R^{2}$ value of 0.81 , showing they are strongly correlated.

Kalt et al. (1999) stored refrigerated raspberries for $8 \mathrm{~d}\left(0{ }^{\circ} \mathrm{C}\right)$. They reported an increase in anthocyanins from $\approx 0.8 \mu \mathrm{mol} \cdot \mathrm{g}^{-1}$ fresh weight to $\approx 1.1 \mu \mathrm{mol} \cdot \mathrm{g}^{-1}$ and no apparent changes in ascorbate or ORAC compared with fresh raspberries. Our results did not find a significant change in ORAC, although total phenolic content increased significantly with refrigerated storage. Mazza and Miniati (1993) reported that raspberry titratable acidity decreases during storage. This decrease in organic acids may be the result of provision of carbon skeletons for the synthesis of phenolics (Kalt et al., 1999). Phenolic synthesis would explain the increases in total phenolics and ORAC found in our experiments, although titratable

Table 1. Cultivar and treatment: mean whole season (five pickings) ORAC and total phenolics of six cultivars of raspberries freshly picked, refrigerated $(7 \mathrm{~d})$, or frozen (3 months). ${ }^{\mathrm{z}}$

\begin{tabular}{lccccccc}
\hline & \multicolumn{3}{c}{ ORAC $(\mu \mathrm{mol} \mathrm{TE} / \mathrm{g})$} & & Total phenolics $(\mathrm{mg}$ of GAE/100 g of fresh weight) \\
\cline { 2 - 4 } Cultivar & Fresh & Refrigerated & Frozen & & Fresh & Refrigerated & Frozen \\
\hline Autumn Bliss & $47.9 \mathrm{~b}$ & $50.3 \mathrm{~b}$ & 41.3 & & $565 \mathrm{~b}$ & $553 \mathrm{ab}$ & 474 \\
Caroline & $33.8 \mathrm{a}$ & $32.7 \mathrm{a}$ & 28.1 & & $444 \mathrm{a}$ & $468 \mathrm{a}$ & 415 \\
Jaclyn & $43.7 \mathrm{ab}$ & $40.6 \mathrm{ab}$ & 38.3 & & $495 \mathrm{ab}$ & $520 \mathrm{ab}$ & 500 \\
Joan J & $44.8 \mathrm{ab}$ & $48.9 \mathrm{ab}$ & 41.8 & & $514 \mathrm{ab}$ & $618 \mathrm{~b}$ & 504 \\
Polana & $36.1 \mathrm{ab}$ & $33.8 \mathrm{ab}$ & 34.5 & & $430 \mathrm{a}$ & $485 \mathrm{a}$ & 406 \\
Polka & $40.4 \mathrm{ab}$ & $45.4 \mathrm{ab}$ & 39.3 & & $437 \mathrm{a}$ & $480 \mathrm{a}$ & 424 \\
\hline
\end{tabular}

${ }^{z}$ Values are the mean of all picking independent determinations. The statistical analysis modeled all of the data together, generating identical ses for each cultivar in each column as follows: ORAC: fresh \pm 2.7 ; refrigerated \pm 3.4 ; frozen \pm 2.8 . Total phenolics: fresh \pm 21 ; refrigerated \pm 25 ; frozen \pm 25 . Different lowercase letters in the same column indicate significant $(P<0.05)$ differences between cultivars for that treatment (there were no significant differences between any cultivars after freezing). No significant differences $(P<0.05)$ were found among fresh, refrigerated, and frozen except for 'Joan J' total phenolics (see text).

$\mathrm{ORAC}=$ oxygen radical absorbance capacity; $\mathrm{TE}=$ Trolox equivalents; $\mathrm{GAE}=$ gallic acid equivalents. 
acidity was not included in our analysis, so this cannot be confirmed. The optimal time to maximize raspberry antioxidant content in both Kalt et al. (1999) and our study appears to be after a refrigerated storage period of 7 to $8 \mathrm{~d}$.

The significant increase in total phenolic content found in the present study after refrigerated storage may be the result of a concentrating of berry phenolics attributable to moisture loss in the refrigerator. To test the extent of evaporation, raspberries were weighed shortly after picking and after $7 \mathrm{~d}$ of refrigerated storage. The berries lost an average of $10.6 \% \pm 0.4 \%$ moisture content. This might be sufficient to remove the significant increase in total phenolic content between fresh and refrigerated raspberries reported in Tables 1 and 2 if adjusted to a fresh picked weight basis. However, others have reported increases in raspberry antioxidant or phenolic content during storage (Kalt et al., 1999; Mazza and Miniati, 1993) under pressureregulated refrigeration. Thus, moisture content is not the only change that occurs during storage, although the two could not be separated in this study as a result of the consumer storage methods used. The results presented are an accurate representation of what a consumer would encounter, and thus the data were not adjusted or reanalyzed to account for moisture loss.

De Ancos et al. (2000) reported no significant change in total phenolics or antiradical efficiency in any cultivar tested after 3 months of frozen storage. Raspberries were initially frozen in liquid nitrogen before being placed in a $-20{ }^{\circ} \mathrm{C}$ freezer. In a follow-up paper by the same research group, González et al. (2003) froze (liquid nitrogen) the same four cultivars of raspberry and stored them at $-20{ }^{\circ} \mathrm{C}$ up to 12 months. After 3 months of frozen storage, they reported a significant decrease in antioxidant capacity for three of the four cultivars, a significant decrease in total anthocyanins in one of the four cultivars, and no change in total phenolic content. We report no significant change between fresh and frozen raspberries in ORAC or total phenolics for any cultivar (Table 1). Thus, with or without liquid nitrogen freezing, 3 months of frozen storage has little effect on antioxidant capacity or phenolic content of raspberries.

Both De Ancos et al. (2000) and Wang and Lin (2000) chose two spring and two fall bearing cultivars of raspberries. They did not compare them directly, although a secondary analysis of their published data was of interest to the present study. The two fall bearing cultivars had a mean total phenolic content $42 \%$ and $16 \%$ higher, respectively, than the two spring cultivars. In González et al. (2003), an antiradical efficiency score for the same cultivars studied in De Ancos et al. (2000) was reported. The fall cultivars had a $94 \%$ higher score than the spring cultivars. The bearing time of raspberry cultivars may be an important factor in antioxidant capacity and phenolic content of raspberries with fall bearing cultivars having higher values.

Differences by harvest time. To determine if there was a significant effect of picking time on differences in ORAC or total phenolics, all cultivars were averaged (Table 3 ). Phenolic content was highest at the beginning and end of the season, whereas ORAC was in general higher later in the season. ORAC and total phenolic content did not always agree, however. This may indicate changes in non-phenolic antioxidants or synergistic/antagonistic interactions of phenolic compounds (Freeman et al., 2010). No other published analyses could be found for comparison that reported collecting raspberries more than once in the same season. The large number of factors that affect a growing season, including weather and pests, and how different cultivars respond to those factors, may also explain the pattern observed.

Comparison with supermarket raspberries. Three cultivars of raspberries were purchased at local supermarkets in the late summer for comparison with the studied cultivars. It appeared from grower information on the packaging that all were grown in California and were thus fall bearing and grown at about the same time of year as the pickings in this study. Cultivar was not indicated on the package, although color, size, and flavor differences of the berries suggested they were three different cultivars. Likewise, picking time could not be determined, although the berries likely were stored under refrigeration during 1 to $3 \mathrm{~d}$ travel to the supermarket and were displayed at room temperature an additional 1 to $3 \mathrm{~d}$. The berries were tested the same day of purchase with no additional storage time, making the comparison most relevant to the refrigerated raspberries in this study. The three ORAC values were $35.0 \pm$ $2.0(\mu \mathrm{mol} \mathrm{TE} / \mathrm{g}$, mean $\pm \mathrm{SD}), 31.3 \pm 2.1$, and $24.6 \pm 2.1$. The total phenolic contents were $357 \pm 14$ (mg of GAE/100 g of fresh weight, mean $\pm \mathrm{SD}$ ), $326 \pm 10$, and $252 \pm 8.2$, in the same order as presented for the ORAC values. Both the ORAC values and total phenolic values were significantly lower than that found for the dry climate cultivars.

Limitations. The raspberries in this study were stored and processed as a consumer might store them, so our results may not be equivalent to berries processed using pressurized refrigeration or liquid nitrogen conditions not commonly available to the consumer. Use of the more controlled conditions could decrease antioxidant or phenolic content on a per gram basis if moisture loss was not a factor or change the antioxidant capacity because smaller ice crystals that form during a faster freezing process are less likely to damage the raspberry tissue, allowing less enzyme/substrate reactions to occur. Because uncontrollable environmental conditions vary from year to year, a similar analysis might yield different results during another harvest season.

\section{Conclusions}

This is the first time six cultivars of primocane raspberries grown under controlled conditions in a hot, dry climate have been compared for their antioxidant and phenolic content. Likewise, this is the first time such berries have been picked across the season and
Table 2. Overall treatment: mean ORAC and total phenolics of six raspberry cultivars and five pickings when freshly picked, refrigerated (7 d), or frozen (3 months). ${ }^{z}$

\begin{tabular}{lcc}
\hline & ORAC & $\begin{array}{c}\text { Total phenolics } \\
(\text { mg of GAE/100 g } \\
\text { of fresh weight })\end{array}$ \\
Treatment & $(\mu \mathrm{mol} \mathrm{TE} / \mathrm{g})$ & $481 \mathrm{a}$ \\
Fresh & $41.1 \mathrm{ab}$ & $520 \mathrm{~b}$ \\
Refrigerated & $42.0 \mathrm{~b}$ & $454 \mathrm{a}$ \\
\hline
\end{tabular}

${ }^{\mathrm{z}}$ Values are the means of all cultivar and picking independent determinations \pm SE. The statistical analysis modeled all of the data together, generating identical SES for each cultivar in each column as follows: ORAC: \pm 1.2 ; total phenolics: \pm 10 . Different lowercase letters in the same column indicate significant $(P<0.05)$ differences among fresh, refrigerated, and frozen.

ORAC $=$ oxygen radical absorbance capacity; $\mathrm{TE}=$ Trolox equivalents; GAE = gallic acid equivalents.

Table 3. Treatment and picking: mean ORAC and total phenolics of freshly picked, refrigerated ( $7 \mathrm{~d}$ ), and frozen (3 months) raspberries by picking, all cultivars combined. ${ }^{\mathrm{z}}$

\begin{tabular}{ccc}
\hline Picking ${ }^{y}$ & $\begin{array}{c}\text { ORAC } \\
(\mu \mathrm{mol} \mathrm{TE} / \mathrm{g})\end{array}$ & $\begin{array}{c}\text { Total phenolics } \\
\text { (mg of GAE/100 g } \\
\text { of fresh weight) }\end{array}$ \\
\hline Fresh & $36.9 \mathrm{a}$ & $573 \mathrm{~b}$ \\
1 & $38.5 \mathrm{a}$ & $363 \mathrm{a}$ \\
2 & ND & $403 \mathrm{a}$ \\
3 & $50.8 \mathrm{~b}$ & $421 \mathrm{a}$ \\
4 & $36.0 \mathrm{a}$ & $655 \mathrm{~b}$ \\
5 & & $476 \mathrm{a}$ \\
Refrigerated & $40.5 \mathrm{a}$ & $411 \mathrm{a}$ \\
1 & ND & $463 \mathrm{a}$ \\
2 & $38.0 \mathrm{a}$ & $557 \mathrm{~b}$ \\
3 & $51.8 \mathrm{~b}$ & $470 \mathrm{~b}$ \\
4 & & $357 \mathrm{a}$ \\
Frozen & $38.4 \mathrm{~b}$ & $391 \mathrm{ab}$ \\
1 & $40.5 \mathrm{bc}$ & $421 \mathrm{ab}$ \\
2 & $45.6 \mathrm{c}$ & \\
3 & $29.5 \mathrm{a}$ & \\
4 & means of all cultivar independent
\end{tabular}

${ }^{\mathrm{z}}$ Values are the means of all cultivar independent determinations. The statistical analysis modeled all of the data together, generating identical SEs for each value in each column as follows: ORAC: \pm 1.4. Total phenolics: \pm 17 . Different lowercase letters in the same column indicate significant $(P<$ $0.05)$ differences between pickings for all cultivars. yPicking numbers refer to raspberries picked 15 and 29 Aug., 12 and 26 Sept., and 10 Oct. 2009.

ORAC $=$ oxygen radical absorbance capacity; $\mathrm{TE}=$ Trolox equivalents; GAE = gallic acid equivalents; $\mathrm{ND}=$ not determined .

tested after two consumer-based storage periods. The stated hypothesis was confirmed for cultivar and harvest time although not for storage conditions. The Autumn Bliss cultivar had the highest overall ORAC and total phenolic content across the season. Only 'Joan $\mathrm{J}$ ' had a significant increase in total phenolic content after refrigeration. Freezing had no significant effects. Berries picked at the beginning or the end of the season had the highest ORAC and total phenolic content. On average, primocane raspberries grown in a hot, dry climate produce a higher antioxidant and phenolic content that berries grown in other climates or grown for supermarket distribution. 


\section{Literature Cited}

Anttonen, M.J. and R.O. Karjalainen. 2005. Environmental and genetic variation of phenolic compounds in red raspberry. J. Food Compost. Anal. 18:759-769.

Cao, G., E. Sofic, and R.L. Prior. 1997. Antioxidant and prooxidant behavior of flavonoids: Structureactivity relationships. Free Radic. Biol. Med. 22: 749-760.

Davalos, A., C. Gomez-Cordoves, and B. Bartolome. 2004. Extending applicability of the oxygen radical absorbance capacity (ORAC fluorescein) assay. J. Agr. Food Chem. 52:48-54.

De Ancos, B., E.M. Gonzalez, and M.P. Cano. 2000. Ellagic acid, vitamin C, and total phenolic contents and radical scavenging capacity affected by freezing and frozen storage in raspberry fruit. J. Agr. Food Chem. 48:45654570.

Dragsted, L.O., M. Strube, and J.C. Larsen. 1993. Cancer-protective factors in fruits and vegetables: Biochemical and biological background. Pharmacol. Toxicol. 72(supp1 1):116135.

Dvaranauskaite, A., P.R. Venskutonis, and J. Labokas. 2006. Radical scavenging activity of raspberry (Rubus idaeus L.) fruit extracts. Acta Aliment. 35:73-83.
Freeman, B.L., D.L. Eggett, and T.L. Parker. 2010 Synergistic and antagonistic interactions of phenolic compounds found in navel oranges. J. Food Sci. 75:C570-C576.

González, E.M., B. de Ancos, and M.P. Cano. 2003. Relation between bioactive compounds and free radical-scavenging capacity in berry fruits during frozen storage. J. Sci. Food Agr. 83:722-726.

Gurovich, L.A. 2008. A model to define fertigation strategies for raspberries, integrating soil water and nutrient availability to cropping objectives. Acta Hort. 777:411-422.

Handley, D.T. 2006. Growing raspberries and blackberries. University of Maine Cooperative Extension. <http://umaine.edu/publications/ $2066 \mathrm{e} />$.

Heinonen, M. 2007. Antioxidant activity and antimicrobial effect of berry phenolics - A Finnish perspective. Mol. Nutr. Food Res. 51:684-691.

Kähkönen, M.P., A.I. Hopia, and M. Heinonen. 2001. Berry phenolics and their antioxidant activity. J. Agr. Food Chem. 49:4076-4082.

Kalt, W., C.F. Forney, A. Martin, and R.L. Prior. 1999. Antioxidant capacity, vitamin C, phenolics, and anthocyanins after fresh storage of small fruits. J. Agr. Food Chem. 47:4638-4644.

Liu, M., X.Q. Li, C. Weber, C.Y. Lee, J. Brown, and R.H. Liu. 2002. Antioxidant and antiproli- ferative activities of raspberries. J. Agr. Food Chem. 50:2926-2930.

Mazza, G. and E. Miniati. 1993. Anthocyanins in fruits vegetables and grains. CRC Press, Boca Raton, FL. p. 105.

Ozgen, M., F.J. Wyzgoskiz, A.Z. Tulio, A. Gazula, A.R. Miller, J.C. Scheerens, R.N. Reese, and S.R. Wright. 2008. Antioxidant capacity and phenolic antioxidants of midwestern black raspberries grown for direct markets are influenced by production site. HortScience 43: 2039-2047.

Swain, T. and E. Hillis. 1959. The phenolic constituents of Prunus domestica. The quantitative analysis of phenolic constituents. J. Sci. Food Agr. 10:63-68.

Verlangieri, A.J., J.C. Kapeghian, S. el-Dean, and M. Bush. 1985. Fruit and vegetable consumption and cardiovascular mortality. Med. Hypoth. 16: 7-15.

Wang, S.Y. and H.-S. Lin. 2000. Antioxidant activity in fruits and leaves of blackberry, raspberry, and strawberry varies with cultivar and developmental stage. J. Agr. Food Chem. 48:140-146.

Wright, S.R., C. Welch, L. Miller, and R.C. Funt. 2001. Raised bed planting demonstration $<$ http://www.ag.ohio-state.edu/ prec/hort/data 2001/raised2001.htm>. 